\title{
An Improved Temperature Compensation Method for Fiber Bragg Grating Pressure Sensor Based on Extreme Learning Machine
}

\author{
Hongying Guo ${ }^{1}$, Jiang Chen ${ }^{2}$, Zhumei Tian ${ }^{1}$, Aizhen Wang ${ }^{1}$ \\ ${ }^{1}$ Department of Electronics, Xinzhou Teachers University, Xinzhou 034000, China \\ ${ }^{2}$ MOE Key Laboratory of Deep Earth Science and Engineering, Sichuan University, Chendu 610065, China
}

Received: March 2, 2021. Revised: August 5, 2021. Accepted: August 25, 2021. Published: August 26, 2021.

\begin{abstract}
According to the problem of the sensor nonlinear changes occur at high temperatures, extreme learning machine model, is presented in this thesis the pressure sensitive grating and removing the temperature of the grating experiment data for training, establish a nonlinear model of wavelength, temperature, predict the experimental temperature, then the temperature data of pressure-sensitive grating the training set of training samples, the nonlinear model, temperature - wavelength prediction test set sample output wavelength, achieve the goal of improved temperature compensation method. The experimental results show that the algorithm can achieve a more ideal temperature compensation effect.
\end{abstract}

Keywords - Fiber Bragg grating(FBG), Pressure Sensor, Temperature compensation, Extreme Learning Machine

\section{INTRODUCTION}

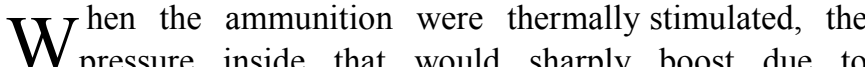
pressure inside that would sharply boost due to temperature variation could cause projectile detonation, which could result in not only ammunition invalidation but furthermore a serious loss of life and property. It is of great practical significance to study the safety and environmental adaptability of the ammunition system by carrying out thermal susceptibility firing experiments of the live ammunition and understanding the changing process of the temperature and pressure in the ammunition body during the heating process. Thermal safety monitoring of ammunition is shown in Figure 1. When external ambient temperature of projectile bodies reaches $1200^{\circ} \mathrm{Cor}$ above, the internal temperature gradually rises and when the internal temperature reaches $300^{\circ} \mathrm{Cor}$ above, projectile bodies would explode. According to the actual application requirements of the projectile body, the detection environment was set as 1) the detection temperature was $100 \sim 300^{\circ} \mathrm{C}$; 2) the pressure measurement range is $0 \sim 50 \mathrm{MPa}$. Under such circumstances, due to the particularity of test medium and strong coupling that caused by various physical fields such as force field, temperature field and vibration field, conventional pressure sensors would reflect the characteristics of variability, coupling and non-linearity, which make traditional electrical, ultrasonic and electromagnetic sensing detection technology do not effectively meet the requirements of pressure detection.

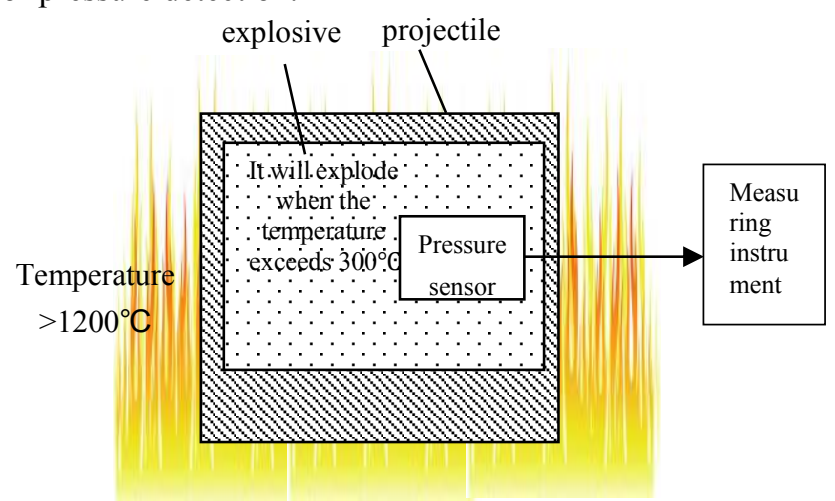

Fig.1 Schematic diagram of solid pressure test under high temperature in an external environment external environment

For the detection of solid pressure, fiber grating pressure sensing technologies can overcome the disadvantages of conventional pressure sensors such as poor stability, poor moisture resistance, and susceptibility to electromagnetic interference in harsh environments; at the same time, the grating sensor is a passive device, which does not need on-site power supplies[1]. In the flammable and combustible environment, or under electromagnetic interference as well as strong radiations, it can exhibit unique advantages. For this reason, a fiber grating solid pressure sensor is specially designed[2] to monitor the internal pressure of the ammunition during the cook-off process of live ammunition, and specifically, realize the real-time pressure monitoring of $0-50 \mathrm{MPa}$. However, Fiber Bragg grating wavelength is sensitive to both temperature and strain, in the process of signal acquisitions and transmissions, due to the interference of the surrounding environment, the multi-physical field coupling of the sensor environment and the accuracy of the demodulation equipment, effective temperature compensation must be carried out[3].

For the temperature compensation of fiber grating pressure sensor, many scholars have conducted in-depth exploration[4,5].Chao Li, Tigang Ning[6], 2016, Reported an 
optical fiber sensor for measurement of liquid level and temperature, the liquid level sensor with dynamic temperature compensation can be easily achieved simultaneous by using the temperature sensing property of FBG. For 10 pm wavelength resolution, the resolution of the sensor is $0.046 \mathrm{~cm}$ and $1.1{ }^{\circ} \mathrm{C}$ in liquid level and temperature, respectively. 2016, Li T., Tan Y. and Zhou Z[7]used two FBGs to achieve double-difference tmperature compensations for the two states of the sensor. Based on the magnetic-coupling and FBG-sensing principles, this operation can be used to achieve non-contact measurements on the vibration of the rotating shaft; when it is used to correct the temperature deviation, in the range of $25 \sim 60^{\circ} \mathrm{C}$, the temperature deviation of the sensor will be reduced to $1.19 \%$.

2017 , they[8] proposed a novel fiber Bragg grating (FBG)

sensing-based acceleration sensor。A decoupling method has been presented with consideration of the thermal expansion of the sensor structure to realize temperature compensation.Experimental results show that the temperature sensitivity is $8.66 \mathrm{pm} /{ }^{\circ} \mathrm{Cwithin}$ the range of $30-90^{\circ} \mathrm{C}$. Yi KUANG, Yongxing GUO[9], 2018, summarizes the packaging methods and corresponding temperature compensation methods of the currently reported focusing especially on fully pasted FBG, pre-stretched FBG with double-end fixed,and metallic packaging. In their reseache, they analyzed the advantages and drawbacks of different packaging methods, which can provide a reference for future researches. 2019 , Ruiya Li , Yuegang Tan, Yiyang Chen[10] came up with a novel method to realize temperature compensation simultaneously for fiber Bragg grating (FBG)-based strain sensor. The decoupling of strain and temperature was implemented theoretically and verified experimentally. The decoupling of strain and temperature was implemented theoretically and verified experimentally.Ma $\mathrm{R}$ [11] in 2018 studied the new compensation method of hull monitoring FBG sensor based on the sensor theory with both FBG temperature self-correction and steel thermal expansion effects correction. The coupled compensation method suitable for hull monitoring sensor is obtained by theoretical derivation. As the comparison, the coupled compensation experiment was carried out. The results show that the relative error under the temperature compensation method is large in the case of drastic strain and temperature changes, and the correction results of the tested method will be closer to the true level. Mengying $\mathrm{T}$ \{Formatting Citation\} in 2018 proposed a novel method based on a digital filtering technique for temperature compensation in fiber Bragg grating sensor systems for monitoring the conditions in electrified pantograph-catenary systems. A Butterworth high-pass filter was designed to reject the temperature-related signal component with a stopband and to capture the real strain in the pass band in frequency domain. Tianliang Li, Chaoyang Shi, Yuegang Tan[13] in 2017 proposed and studied a novel diaphragm-type fiber Bragg grating (FBG) vibration sensor with a small mass and an excellent sensitivity through the use of the transverse property of a tightly suspended optical fiber.The temperature compensation Compared with the existing diaphragm-enabled FBG vibration sensors, the proposed sensor enables to support the easy implementation of distributed measurement, and the small mass allows for detection on mass-sensitive structures.

These temperature compensation methods can be classified into three types: 1) Referring to the FBG method, two FBGs FBG1 and FBG2 are arranged inside the packaging structure of the sensor. FBG1 is the detection FBG that is affected by the environmental temperature and the deformation of the packaging model at the same time;FBG2 is a temperature compensated FBG, placed in the same temperature field with FBG1.However, when FBG2 is laid out, one end is usually a free end, the deformation of the sensor packaging structure will not generate strain on it, and its output is only affected by the change of temperature. The two FBGs are in the same temperature field, and the change information of the ambient temperature of the sensor can be obtained through FBG2, and then the influence of temperature on the output of the pressure-sensitive FBG ( FBG1 ) can be obtained.2)

Differential setting method, in which method, two FBGs are arranged in different positions of the sensor sensitive parts. These two FBGs are arranged close to each other and in the same environment, so they have the same temperature change. Therefore, the influence of temperature can be eliminated by taking the difference value of the wavelength change of the two FBGs.3) Soft compensation method. The soft compensation method processes the collected data by selecting an appropriate algorithm to eliminate the impact of temperature.Most of the above references use differential compensation method. The advantage of this method is that in the detection process, the strain sizes of the two FBGs are different, and one of the two FBGs is stretched and the other is compressed, so the strain difference between the two FBGs is large, and the detection sensitivity can be obtained.The disadvantage is that it requires two FBGs to have exactly the same temperature sensitive characteristics, which is difficult to achieve in specific operation.In addition, it also increases the complexity of sensor packaging and puts forward higher requirements for the manufacturing process.The ambient temperature of this test is 300 . Various unpredictable problems will occur in the complex process at high temperature.So in this paper, the reference FBG method and soft compensation method are used together.

In recent years, many scholars have combined grating sensing technology with various signal processing methods (such as: Wavelet transform[14], BP neural network[15,16], coupled neural network modeling[17,18], Hilbert-yellow transform[19,20], Duffing chaotic oscillator model, etc., are combined to carry out damage identification, fault location, real-time monitoring and other studies[21-23], and have been widely used in many fields [24-26]. Nevertheless, because of the particularity of the environment inside the ammunition, there are few researches on the thermal safety detection 
environment of ammunition using grating sensing technology and signal processing method and the temperatures have a significant impact on pressure test results, which ask for an attention on temperature compensation.

However, the research on the thermo-safety detection environment of the ammunition is still less. The particularity on the internal environment of the ammunition makes the temperature have significant impacts on the detection results of pressure, so we must pay attention to temperature compensation. This paper abstracts the compensation problems of pressure sensors under high temperature as pattern recognition ones in machine learning, and establishes nonlinear recognition models integrated with ELM to provide accurate and fast recognition models for solving these problems. Based on the temperature data of pressure-sensitive gratings and temperature compensation gratings in pressure sensors, the training set and test set are formed respectively, and based on the two training sets, the nonlinear recognition model is further established to recognize the output wavelength in the test sample.

\section{TEMPERATURE EXPERIMENT PLATFORM}

\section{A. Sensor Structure}

The fiber grating solid pressure sensor is composed of a pressure-bearing diaphragm, a sensor housing and an upper cover of stainless steel. The base materials used for the two parts are the same. The pressure-bearing diaphragm is composed of a circular elastic diaphragm and a solid pressure grating fixed column. The fixed column, the elastic diaphragm and the sensor housing are processed in one to reduce the problem of stress concentration or unbalanced stress. The sensor housing and the upper cover of stainless steel adopt threaded connections. The pressure diaphragm is used as the elastic element of the sensor to sense the external pressure, and two symmetrical fixing columns are set on the pressure diaphragm to fix the two ends of the solid pressure-sensitive grating FBG1. The solid pressure is converted into measurable strains by the fiber grating through the circular elastic diaphragm. The specific structure is shown in Figure 2.

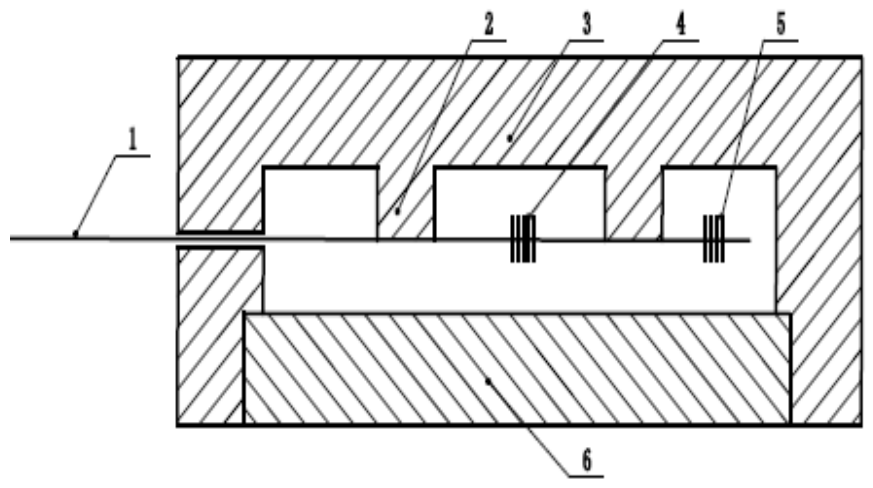

Fig. 2 Cross-sectional view of the structure of the fiber grating pressure sensor

The package structure of the sensor should be able to convert the external pressure into the strain of the FBG, i.e. the external pressure is converted into the stretching or compression of the grating. In order to achieve this goal, two fixed columns are arranged symmetrically and vertically inside the pressure diaphragm of the sensor to fix the two ends of the grating. When pressure is applied to the diaphragm, it will cause a downward micro-deformation of the diaphragm .This deformation will drive the two fixed columns to produce deformation in opposite directions, which will pull the grating to produce strain on the grating. Select the incubator, fiber demodulator (SM125 produced by MOI Company, demodulation range of the wavelength: $1510 \sim$ $1590 \mathrm{~nm}$, accuracy: $1 \mathrm{pm}$ ), pressure sensor samples under high temperature and computer to build a temperature calibration experiment platform. Set the temperature of the incubator to $100^{\circ} \mathrm{C}$, keep it warm for 4 hours, increase the temperature by $20^{\circ} \mathrm{C}$, and keep circulating until the temperature rises to $300^{\circ} \mathrm{C}$. At $20^{\circ} \mathrm{C}$ intervals, the temperature is lowered to $100^{\circ} \mathrm{C}$, and each temperature state is kept for 4 hours. Record the output wavelength of pressure-sensitive gratings and temperature compensated gratings.

\section{B. Traditional Temperature Compensation Methods of} Pressure Grating Sensors

Two fiber gratings are arranged inside the sensor package at the same time to compensate the temperature of the fiber pressure grating sensors. One of them is the pressure-sensitive grating. The two sides of the pressure sensitivity are respectively bonded to two fixed posts to measure the external pressure. The change of pressure and temperature will affect the pressure-sensitive grating. The other is the temperature-compensated grating, which is arranged inside the sensor, and one of the ends is free. The deformation of the sensor package structure will not affect compensation grating of the temperature, and the output of which is only affected by the temperature, which can be used to measure the temperature around the sensor. Two gratings are arranged inside the sensor, which can be considered to be in the same temperature field.

The effects of the temperature and strain on the output of the fiber grating are independent and linearly superimposed. The output of the grating is at $300^{\circ} \mathrm{C}$ and there are no multi-field coupling effects within the elastic deformation range. Under the combined action of the temperature and strain, the output change of the grating can be expressed as

$$
\Delta \lambda_{\mathrm{B}}=\alpha_{\varepsilon} \Delta \varepsilon+\alpha_{\mathrm{T}} \Delta \mathrm{T}
$$

In Equation (1), $\alpha_{\varepsilon}$ is the strain sensitivity coefficient of the grating, $\alpha_{\mathrm{T}}$ is the temperature sensitivity coefficient of the grating, and $\Delta \varepsilon$ is the strain occurring on the grating, $\Delta \mathrm{T}$ is the external temperature variation. In this design, the wavelength changes of the two gratings are as follows

$$
\Delta \lambda_{\mathrm{B} 1}=\alpha_{\varepsilon 1} \Delta \varepsilon+\alpha_{\mathrm{T} 1} \Delta \mathrm{T}
$$




$$
\Delta \lambda_{\mathrm{B} 2}=\alpha_{\mathrm{T} 2} \Delta \mathrm{T}
$$

Then

$$
\Delta \varepsilon=\frac{\Delta \lambda_{\mathrm{B} 1}-K \Delta \lambda_{\mathrm{B} 2}}{\alpha_{\varepsilon 1}}
$$

In Equation (4), $\mathrm{K}=\alpha_{\mathrm{T} 1} / \alpha_{\mathrm{T} 2}$. Because two fiber gratings are in the same temperature field, the temperature changes induced by the two gratings are the same. $\Delta \mathrm{T}_{2}$ can be calculated from Equation (3) and substituted into Equation (2) to eliminate the wavelength shift caused by the temperature change of the pressure sensitive grating, thus the strain caused by the external pressure can be obtained from Equation (4). Finally, according to the corresponding relationship between the structure of the sensor package and its related parameters, the external pressure-changing value is calculated.

\section{Temperature Test of Fiber Grating Solid Pressure} Sensor

When the ambient temperature of the sensor rises, sensor package structure would lead to thermal expansion, which would cause grating strain and poor performance of grating outputs. In order to solve the problem, the temperature test devices of the sensors are set up, as shown in Figure 3.

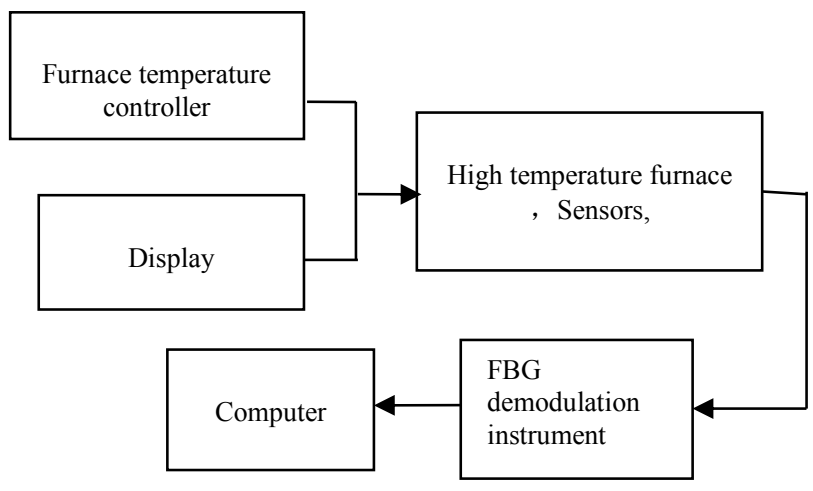

Fig. 3 Device diagram of temperature experiment

The sensor was put into a calorstat and three rounds of experiments on high temperature $\left(300^{\circ} \mathrm{C}\right)$ and room temperature $\left(20^{\circ} \mathrm{C}\right)$. Afterwards, a gradually warming mode was carried out from $100^{\circ} \mathrm{C}$ to $300^{\circ} \mathrm{C}$, with an increased temperature of $20^{\circ} \mathrm{C}$ per time. Then comes the temperature reduction and during the cooling process, the temperature starts from $300^{\circ} \mathrm{C}$ to $100^{\circ} \mathrm{C}$, with an decreased temperature of $20^{\circ} \mathrm{C}$ each time. After thermal insulation for $4 \mathrm{hrs}$ at each temperature point, the outputs of pressure-sensitive grating FBG1 and temperature-compensated grating FBG2 of the sensor were recorded at an interval of 10 minutes and the output value of the point after 15 times records was the average value. The above experiment was carried out for 3 times to record the grating output data and draw the temperature-output wavelength diagram, as shown in Figure 4 and Figure 5. The average output at each temperature point was taken as the calibration value for data analysis and processing and the sensitivity of the pressure-sensitive grating FBG1 and temperature-compensated grating FBG2 of the sensor to the temperature response could be recorded.

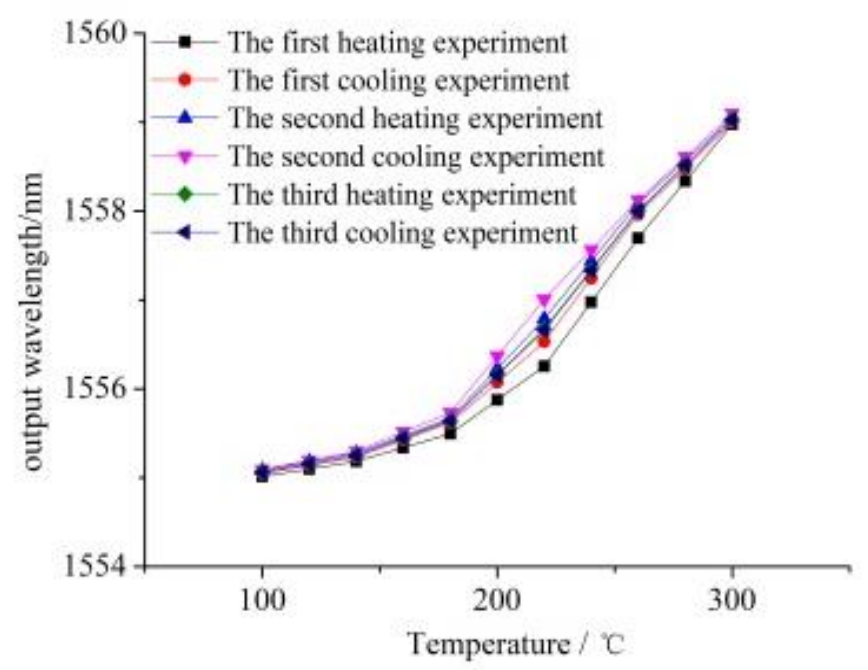

Fig. 4 Temperature experimental data graph of pressure sensitive grating

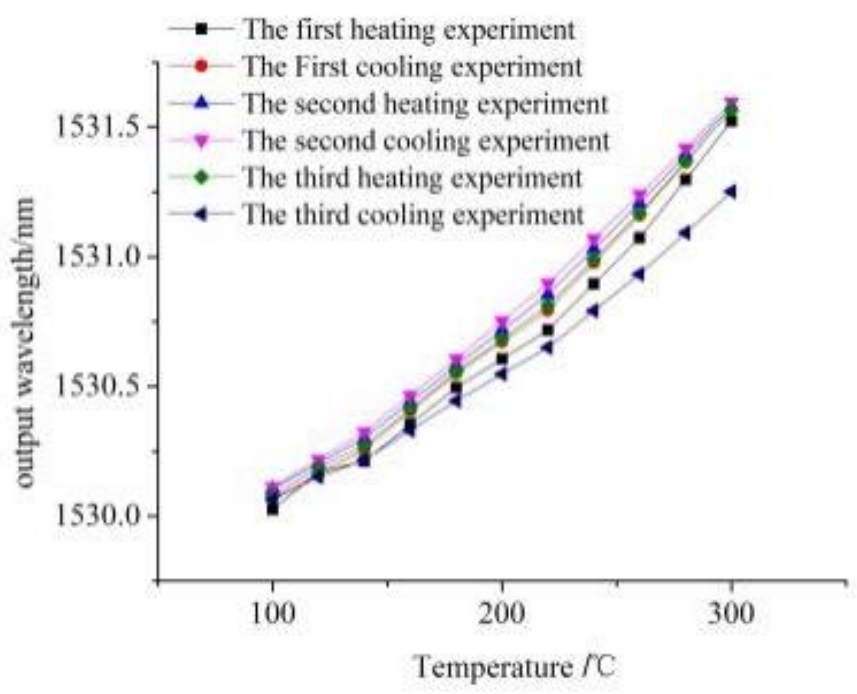

Fig. 5 Temperature experimental data graph of temperature compensated grating

As can be seen from Figure 4 and Figure 5, the center offset of the sensor's spectral line presents obvious nonlinear characteristics with the increase of temperature under high temperature environment, especially the nonlinear characteristics of the pressure-sensitive grating. The main reason for this phenomenon is that the thermal expansion coefficient of the packaging material changes with the temperature. In a wide range of temperature changes, the thermal expansion of the sensor leads to a certain nonlinear change in the distance between the two fixed columns, and then makes the center wavelength of the varistor-sensitive grating show obvious nonlinear. By substituting the experimental data into the temperature compensation formula, the pressure measurement deviation curves at different temperatures can be calculated when the pressure is 0 , as it is shown in Figure 6. 


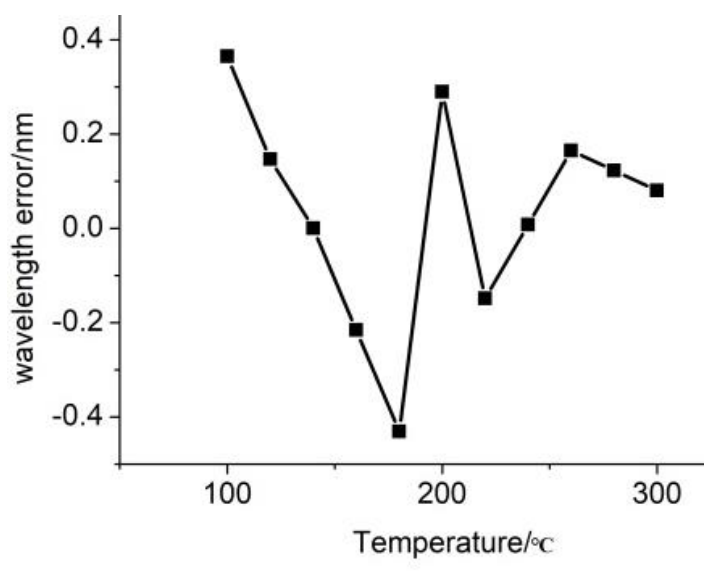

Fig. 6 Output error curve

\section{COnstruction of DAta Processing Models}

Adopt the idea of one-time learning to ELM. When learning the model, we only need to choose the appropriate number of hidden layer neurons. The thresholds of the hidden layer neurons as well as the connection weight between the hidden layers and the input layer can be randomly generated, and there is no need to adjust the weight of the output layer artificially during the entire training process. By using the least square method, the whole learning process can be completed at one time without iterations.

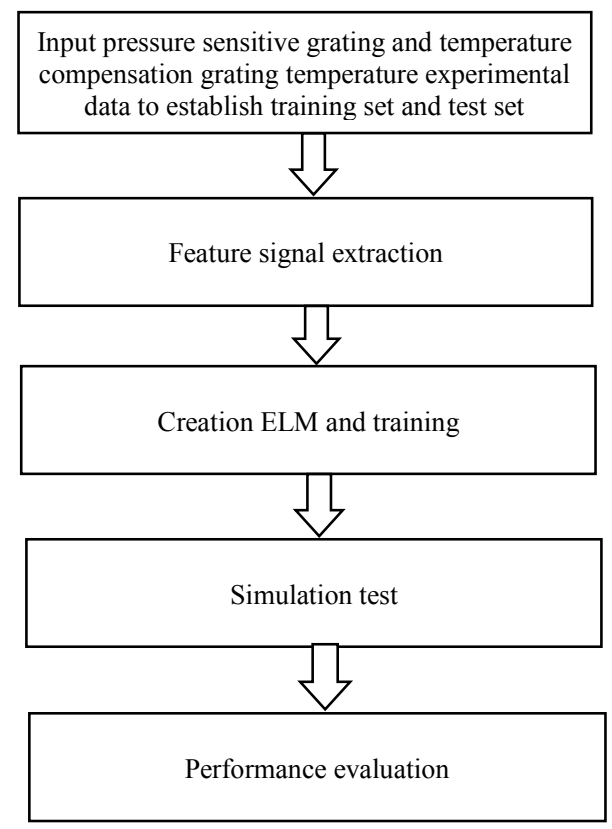

Fig.7 Work steps of ELM model

Therefore, compared with the traditional single-hidden-layer feed-forward neural network training method, ELM has stronger learning abilities, fast learning speeds and good generalization performances. Besides, ELM can approximate complex nonlinear functions. When establishing the model, we only need to create ELM as well as train and simulate according to the requirements of the problem to be solved. The process is shown in Figure 7.

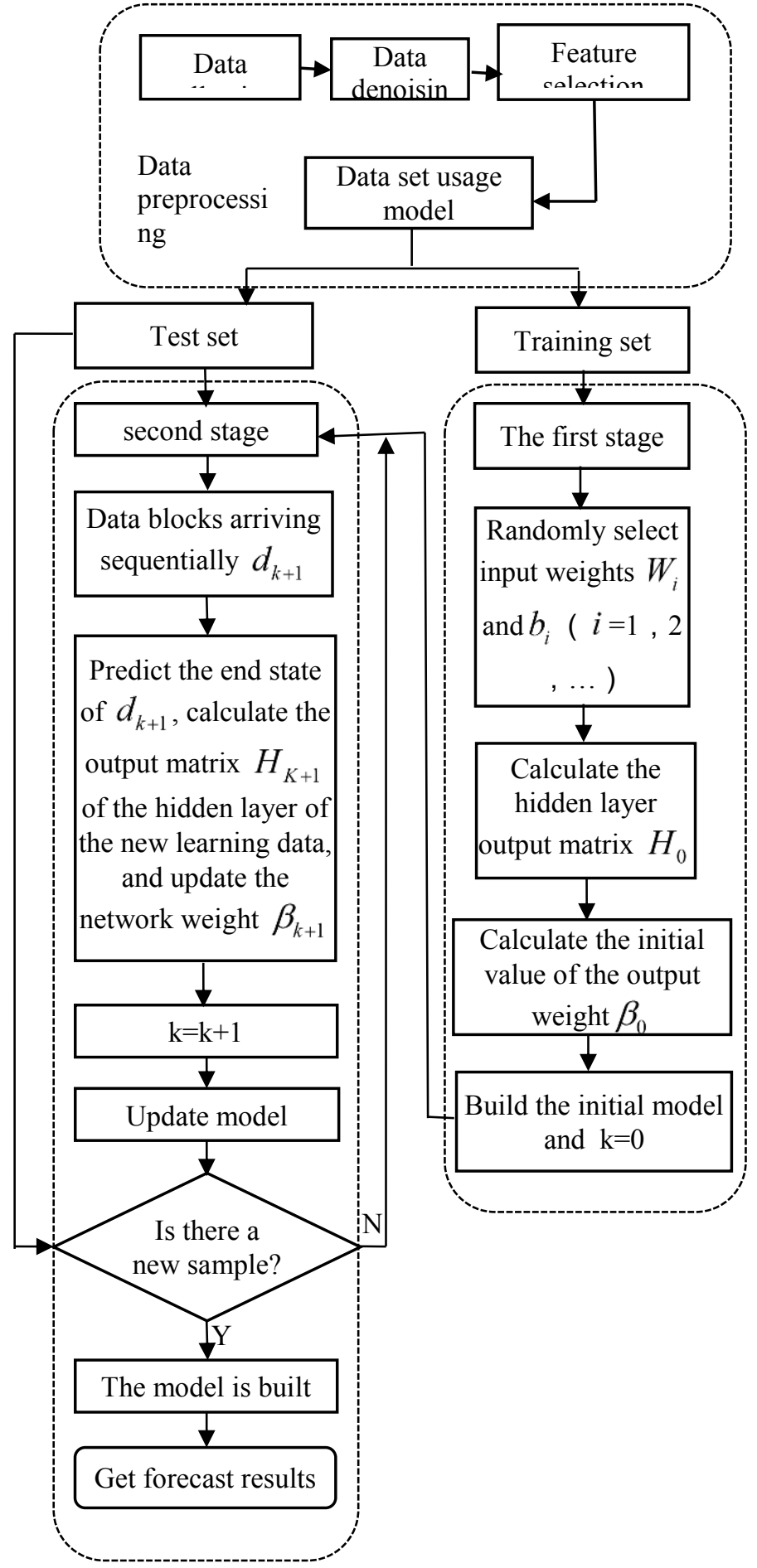

Fig. 8 Flow chart of the prediction process of the ultimate learning machine

The ELM algorithm includes two phases: initialization phase and online continuous learning phase: •

\section{Step 1 Initialization :}

Given the training set $D$ and the number of hidden layer nodes $L$, select part of the data set $D_{0}=\left\{\left(x_{i}, t_{i}\right), i=1,2, \cdots, N_{0}\right\}$, where $N_{0}$ represents the 
selected initial number, and $N_{0} \geq L$.The input weight $w_{i}(i=1,2, \cdots, L)$ and threshold $b$ of hidden layer nodes are randomly selected. •

1) Calculate the hidden layer output matrix ${ }_{H_{0}} \cdot$ •

2) According to the target output ${ }_{T_{0}=\left(t_{1}, t_{2}, \cdots, t_{N_{0}}\right)^{T}}$, calculate the initial output weight $\beta^{0}, \beta^{0}=P_{0} H_{0}^{T} T_{0}$, where $P_{0}=\left(H_{0}^{T} H_{0}\right)^{-1}$

- 3) make $k=0^{\cdot}$

Step 2 Online continuous learning :

1) Assume that the data block added in the $k+1$ step is $\Omega_{k+1}=\left\{\left(x_{i}, t_{i}\right)\right\} \sum_{j=0}^{k+1} N_{j}$, where $N_{K+1}$, represents the number of data added in the $k+1$ step; $\cdot$

2) Compute the hidden layer output matrix $H_{k+1}$ of the newly learned data

3) make $T_{k+1}=\left(t_{\left(\Sigma_{j=0}^{k} N_{j}\right)}, \cdots, t_{\left(\sum_{j=0}^{k+1} N_{j}\right)}\right)^{T}$

4) Calculate the output weight $\beta^{k+1}=\beta^{k}+P_{k} H_{k+1}^{T}\left(T_{k+1}-H_{k+1} \beta^{k}\right)$, $P_{k+1}=P_{k}-P_{k} H_{k+1}^{T}\left(I+H_{k+1} P_{k} H_{k+1}^{T}\right)^{-1} H_{k+1} P_{k}, I$ is the identity matrix

5 ) Let $k=k+1$ and return to Step 2

The algorithm is tested on the temperature data of self-test sensors, and the PC used in the experiment is basically configured as Intel(R)Core(TM)i7-4790CPU@3.60GHz, with a 8.00GB RAM and a 64-bit operating system. The experimental software is MATLAB R2014a. The training sample set and test sample set are generated separately from the standard data set at a ratio of 7:4, and the ELM code is input for training. The training process is shown in Figure 8.

In the prediction process experiment of the ELM, train the sample set of the temperature experimental data for the temperature compensation grating first (including the experimental temperature and the central reflection wavelength of the temperature compensation grating), complete the establishment of the temperature-wavelength model, and then use the model to predict the test sample set (predict the experimental temperature) and compare it with the experimental temperature. The results of multiple comparisons are shown in Figure 9.

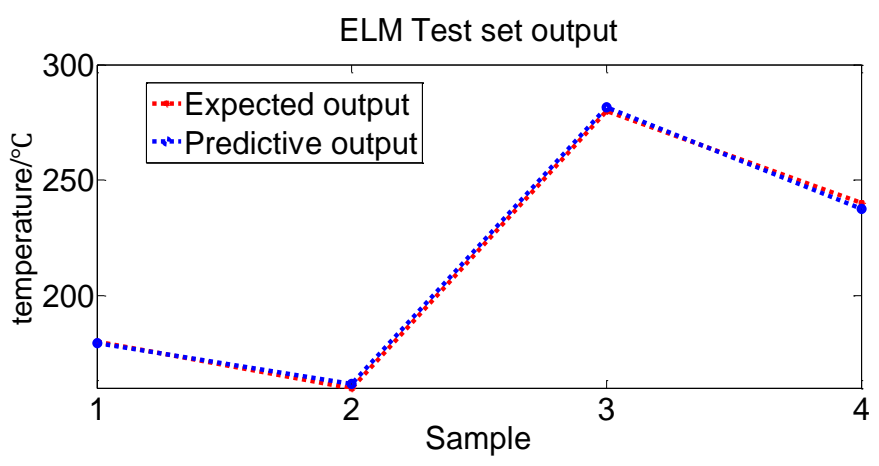

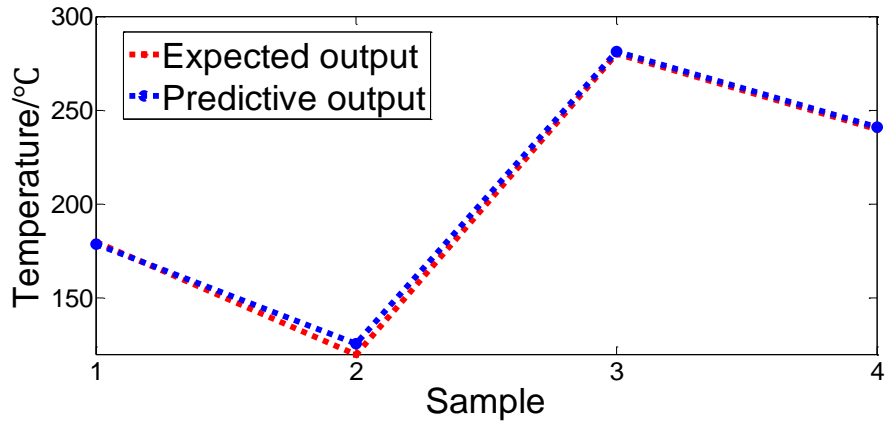

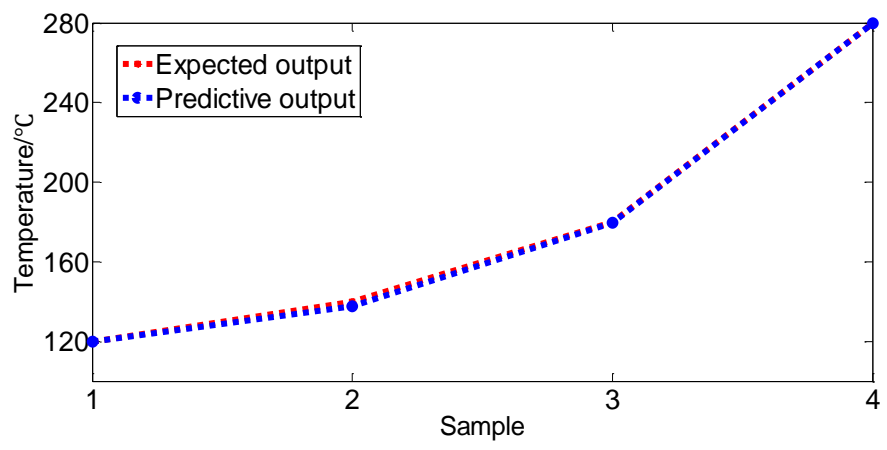

Fig.9 Experimental comparisons between the predicted temperature output and the actual temperature

Output and show the temperature predicted by the ELM models, and compare it with the actual temperature and the one calculated using the sensor temperature sensitivity. As shown in Fig 10, the temperature in the table represents the actual one of the experimental environment, and the predicted temperature represents the output one predicted by the ELM model. The sensor is calibrated by the experimental data to get the temperature sensitivity, and then the corresponding temperature is calculated. Deviation 1 represents the absolute one of the ELM model in predicting the temperature, and deviation 2 represents the temperature one calculated by sensor sensitivity. By comparison, it can be seen that the prediction deviation of the ELM model is far less than the calculation deviation, which shows that the ELM can improve the temperature output accuracy of the grating effectively, so as to improve the temperature compensation effect of the sensor. 


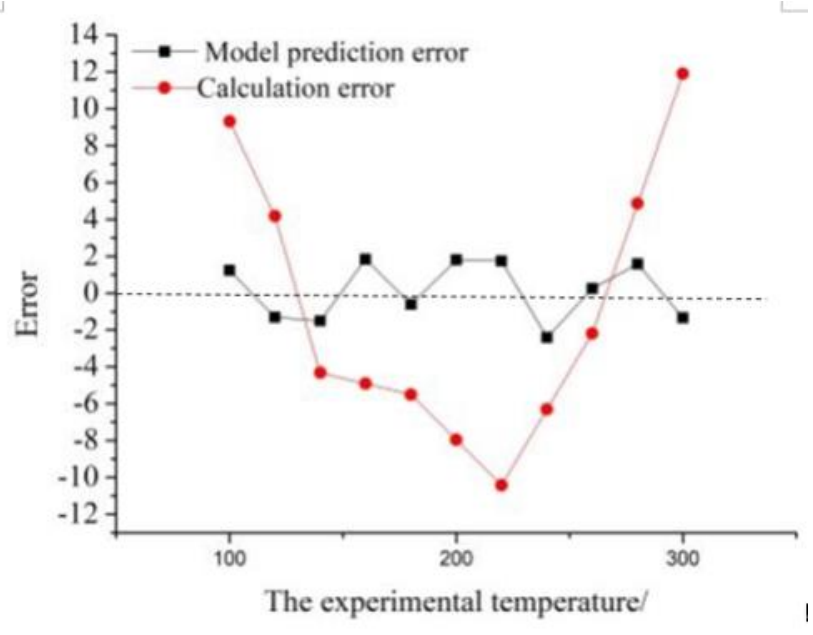

Fig. 10 Error contrast between the predicted temperature made by extreme learning machine and the calculated temperature offered by sensor sensitivity

In Figure 10, the dotted line is the accurate location of temperature prediction. The closer to the dotted line is, the more accurate the temperature prediction is.The accurate identification of the temperature is the basic guarantee for the subsequent temperature compensation to achieve good results.It can be seen from Figure 10 that the black points are closer to the dotted line than the red points, that is to say, the ELM algorithm has more advantages in detecting the environmental temperature and predicting.

Train the sample set of the pressure-sensitive grating temperature data (including experimental temperature and the center reflection wavelength of the pressure-sensitive grating), and establish a temperature-wavelength nonlinear model. The pressure-sensitive grating and the temperature-compensated grating are in the same temperature field, so the predicted temperature in step 1 is input into the model to predict the output wavelength of the test sample set, and compare it with the central reflection wavelength of the pressure-sensitive grating. The output result of multiple training is shown in Fig. 11.

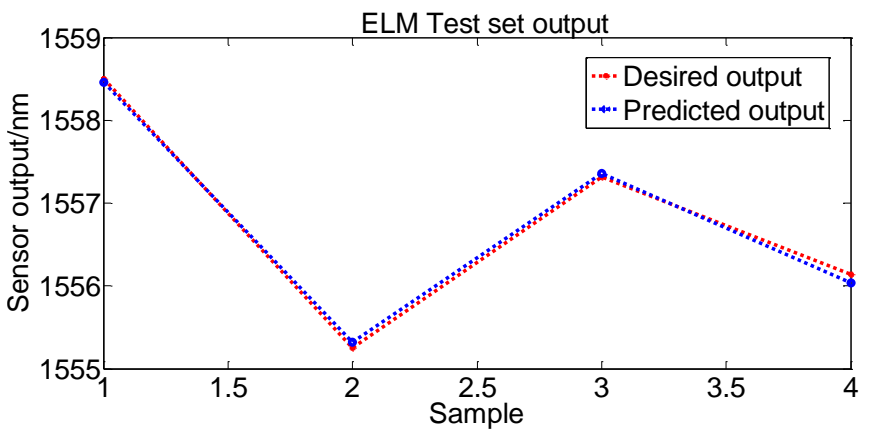

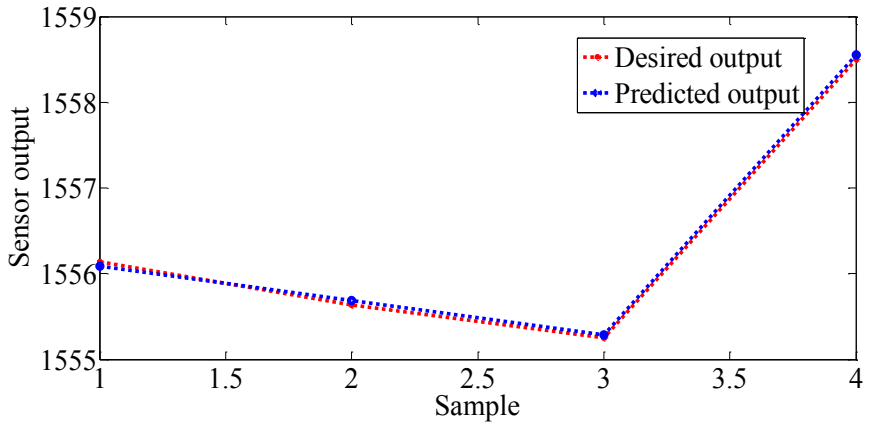

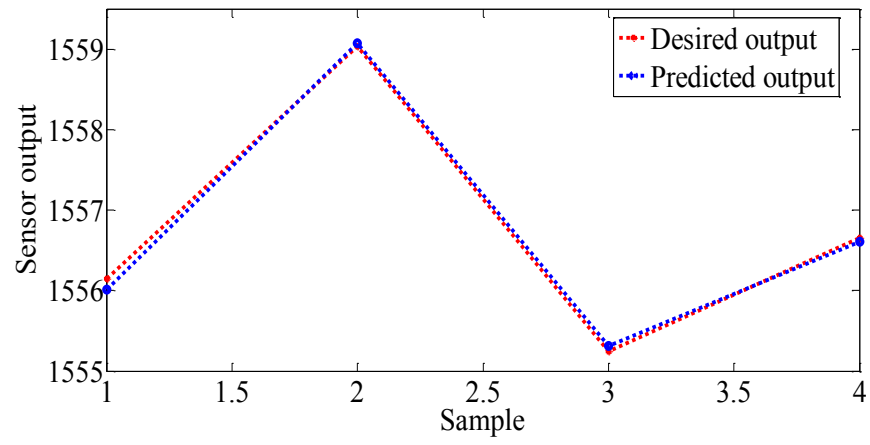

Fig.11 Comparison between high-temperature output prediction and actual output of pressure sensitive grating

Output the prediction result, and compare it with the traditional temperature compensation method, as it is shown in Fig 12.

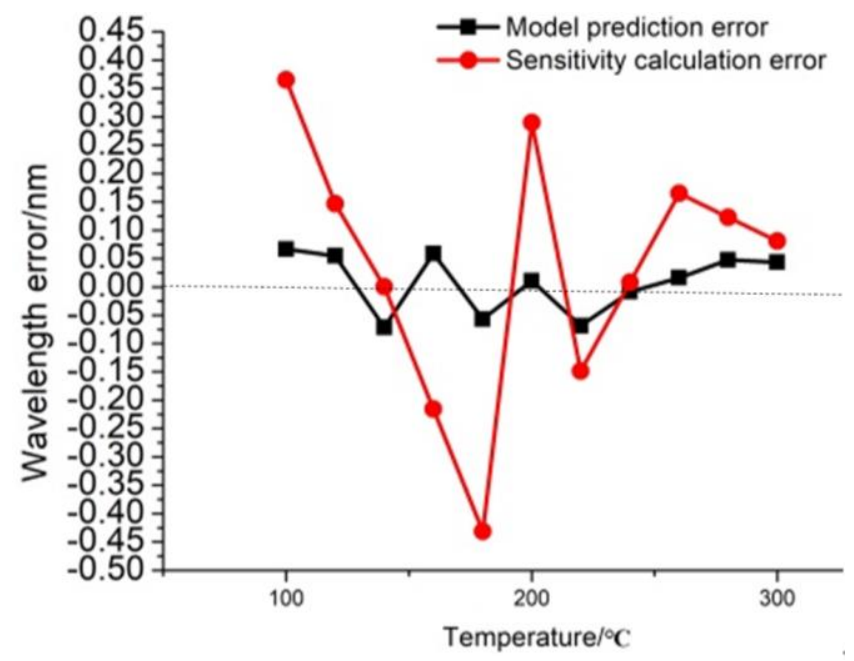

Fig. 12 Comparative analysis of wavelength prediction of pressure sensitive grating

In Figure 12, the dotted line is the ideal position for temperature compensation. The closer it is to the dotted line, the smaller the error of temperature compensation will be, indicating the better the effect of temperature compensation. It can be seen from Figure 12 that the black points are significantly closer to the dotted line than the red points, indicating that the ELM algorithm is introduced for data processing and has achieved better warming effect. The extreme learning machine model was used to predict the influence of temperature on the pressure sensitive grating under different temperature environments. The error was 
improved significantly, and the maximum output error of the pressure sensitive grating was reduced from $0.46 \mathrm{~nm}$ to $0.05 \mathrm{~nm}$. The results show that the extreme learning machine model is selected to process the experimental data of the temperature calibration of the fiber Bragg grating pressure sensor, which can better solve the problem of temperature compensation of the high temperature Bragg pressure sensor and improve the detection accuracy and linearity.

\section{CONCLUSION}

In the detection environment of the cook-off test, the ammunition temperature varies widely, and the temperature compensation deviation of the pressure grating sensor is large. To solve these problems, this paper proposes a signal processing algorithm based on the ELM model to realize the accurate temperature prediction of the temperature compensation grating, and makes precise inferences about the output wavelength of the pressure sensitive grating. The maximum error is reduced from $0.46 \mathrm{~nm}$ to $0.07 \mathrm{~nm}$, and the FBG output signal can be accurately predicted in high temperature environment, which provides a strong support for improving the temperature compensation method in high temperature environment.

\section{REFERENCES}

[1] Lee B. Review of the present status of optical fiber sensors. Optical Fiber Technology 2003; 9(2): 57-79.

[2] Guo HY, Wang ZB, Li HY. Development and Commissioning of High Temperature FBG Solid Pressure Sensors. Journal of Sensors 2018; 8: 1-9.

[3] Li F, Du Y, Zhang W, Li F. Fiber Bragg grating soil-pressure sensor based on dual L-shaped levers. Optical Engineering 2013; 52(1): 014403.

[4] Dvoynishnikov S V, Rahmanov V V, Pavlov VA, Krotov $\mathrm{S}$ V. Comprehensive temperature control of a hot metal rolling thickness measurement system. 2020;15: 191195..

[5] Pilakkat D, Kanthalakshmi S. Study of the Importance of MPPT Algorithm for Photovoltaic Systems under Abrupt Change in Irradiance and Temperature Conditions. 2020; 15: 8-20.

[6] Li C, Ning T, Zhang C, et al. Liquid level measurement based on a no-core fiber with temperature compensation using a fiber Bragg grating. Sensors \& Actuators: 2016; 245: 49-53.

[7] Li T, Tan Y, Zhou Z, Zheng K. A non-contact FBG vibration sensor with double differential temperature compensation. Optical Review 2016; 23(1): 26-32.

[8] Li T, Tan Y, Han X, Zheng K, Zhou Z. Diaphragm Based Fiber Bragg Grating Acceleration Sensor with Temperature Compensation. 2017.

[9] Kuang Y, Guo Y, Xiong L, Liu W. Packaging and Temperature Compensation of Fiber Bragg Grating for Strain Sensing: A Survey. 2018; (May).

[10] Li R, Tan Y, Chen Y, Hong L, Zhou Z. Optical Fiber Technology Investigation of sensitivity enhancing and temperature compensation for fiber Bragg grating ( FBG ) -based strain sensor. Optical Fiber Technology 2019; 48(November 2018): 199-206.

[11] Ma R, Wang Y. INVESTIGATION ON TEMPERATURE COMPENSATION OF FIBER BRAGG GRATING. 2018: 1-6.

[12] Tan M, Zhou N, Cheng Y, Wang J. A temperature-compensated fiber Bragg grating sensor system based on digital filtering for monitoring the pantograph - catenary contact force. 2018; $\mathbf{0}(0): 1-14$.

[13] Li T, Shi C, Tan Y, Li R, Zhou Z, Ren H. A diaphragm type fiber Bragg grating vibration sensor based on transverse property of optical fiber with temperature compensation. 2016; (c): 1-8.

[14] Yeh C, Tsai N, Zhuang Y, Chow C, Liu W. Fault Self-Detection Technique in Fiber Bragg Grating-Based Passive Sensor Network. 2016; 16(22): 8070-8074.

[15] Panopoulou A, Loutas T, Roulias D, Fransen S, Kostopoulos V. Acta Astronautica Dynamic fiber Bragg gratings based health monitoring system of composite aerospace structures \$. Acta Astronautica 2011; 69(7-8): 445-457.

[16] Sun a. Study of simultaneous measurement of temperature and pressure using double fiber Bragg gratings with polymer package. Optical Engineering 2005; 44(3): 034402.

[17] Roveri N, Carcaterra A. Phase-based FBG detectors for self-diagnosis of marine vehicles structures. 2014; (May).

[18] Dvorak M, Kabrt M, Ruzicka M. The use of Fiber Bragg Grating Sensors During the Static Load Test of a Composite Wing Structure. 2014; 486: 102-105.

[19] Soares CG. Volume 1 Sustainable Maritime Transportation and Exploitation of Sea Resources.

[20] Frieden J, Cugnoni J, Botsis J, Gmür T. Low energy impact damage monitoring of composites using dynamic strain signals from FBG sensors - Part II : Damage identification. Composite Structures 2012; 94(2): $593-$ 600.

[21] Poeggel S, Tosi D, Duraibabu D, Leen G, Mcgrath D, Lewis E. Optical Fibre Pressure Sensors in Medical Applications. 2015: 17115-17148.

[22] Jin J, Lin S, Ye X. Optik FBG sensor network for pressure localization of spacecraft structure based on distance discriminant analysis. Optik - International Journal for Light and Electron Optics 2014; 125(1): 404-408.

[23] Kanellos GT, Papaioannou G, Tsiokos D, Nianios G, Pleros N. Two dimensional polymer-embedded quasidistributed FBG pressure sensor for biomedical applications. 2010; 18(1): 583-589.

[24] Miyauchi Y, Ishizawa H, Koyama S, Sato S. Verification of the Systolic Blood-pressure Measurement Principle by FBG Sensors. 2012: 619-622.

[25] Miyauchi Y. Basic Experiment of Blood-pressure Measurement which Uses FBG Sensors.

[26] Poeggel S, Duraibabu D, Lacraz A, Kalli K. Multi FBG Femtosecond laser inscription in FPI based pressure sensors for temperature distribution. 2015; 9634: 1-4.

\section{Creative Commons Attribution License 4.0 (Attribution 4.0 International, CC BY 4.0)}

This article is published under the terms of the Creative Commons Attribution License 4.0

https://creativecommons.org/licenses/by/4.0/deed.en_US 